\title{
Young Stellar Populations around SN 1987A
}

\section{Citation}

Panagia, Nino, Martino Romaniello, Salvatore Scuderi, and Robert P. Kirshner. 2000. "Young Stellar Populations around SN 1987A." The Astrophysical Journal 539 (1): 197-208. https:// doi.org/10.1086/309212.

\section{Permanent link}

http://nrs.harvard.edu/urn-3:HUL.InstRepos:41399861

\section{Terms of Use}

This article was downloaded from Harvard University's DASH repository, and is made available under the terms and conditions applicable to Other Posted Material, as set forth at http:// nrs.harvard.edu/urn-3:HUL.InstRepos:dash.current.terms-of-use\#LAA

\section{Share Your Story}

The Harvard community has made this article openly available.

Please share how this access benefits you. Submit a story.

\section{Accessibility}


The AstrophysicAl Journal, 539:197-208, 2000 August 10

\title{
YOUNG STELLAR POPULATIONS AROUND SN 1987A ${ }^{1}$
}

\author{
NinO PANAGIA ${ }^{2}$ \\ Space Telescope Science Institute, 3700 San Martin Drive, Baltimore, MD 21218 \\ MARTINO ROMANIELlO \\ European Southern Observatory, Karl-Schwarzschild-Strasse 2, D-85748 Garching bei München, Germany
}

SALVATORE SCUDERI

Osservatorio Astrofisico di Catania, Via Santa Sofia 78 I-95125, Catania, Italy

AND

ROBERT P. KIRSHNER

Harvard-Smithsonian Center for Astrophysics, 60 Garden Street, Cambridge, MA 02138

Received 1999 November 24; accepted 2000 March 2

\begin{abstract}
We present the first results of a study of the stellar population in a region of 30 pc radius around SN 1987A, based on an analysis of multiband Hubble Space Telescope (HST) WFPC2 images. The effective temperature, radius and, possibly, reddening of each star were determined by fitting the measured broadband magnitudes to the ones calculated with model atmospheres. In particular, we have determined effective temperatures and bolometric luminosities for 21,995 stars, and for a subsample of 2510 stars we also determined individual reddening corrections. In addition, we have identified all stars with $\mathrm{H} \alpha$ equivalent widths in excess of $8 \AA$, a total of 492 stars. An inspection of the H-R diagram reveals the presence of several generations of young stars, with ages between 1 and $150 \mathrm{Myr}$, superposed on a much older field population (0.6-6 Gyr). A substantial fraction of young stars with ages around 12 Myr make up the stellar generation coeval to SN 1987A progenitor. The youngest stars in the field appear to be strongline T Tauri stars, identified on the basis of their conspicuous ( $W_{\text {eq }}>8 \AA$ ) $\mathrm{H} \alpha$ excesses. This constitute the first positive detection of low-mass (about 1-2 $M_{\odot}$ ) pre-main-sequence (PMS) stars outside the Milky Way. Their positions in the H-R diagram appear to require that star formation in the LMC occurs with accretion rates about 10 times higher than in the Milky Way, i.e., $\sim 10^{-4} M_{\odot} \mathrm{yr}^{-1}$. SN 1987A appears to belong to a loose, young cluster $12 \pm 2 \mathrm{Myr}$ old, in which the slope of the present mass function is almost identical to Salpeter's, i.e., $\Gamma=d \log N / d \log M \simeq-1.25$ for masses above 3 $M_{\odot}$, but becomes much flatter for lower masses, i.e., $\Gamma \simeq-0.5$. On a large scale, we find that the spatial distributions of massive stars and low-mass PMS stars are conclusively different, indicating that different star formation processes operate for high- and low-mass stars. This results casts doubts on the validity of an initial mass function (IMF) concept on a small scale (say, less than $10 \mathrm{pc}$ ). Moreover, it appears that a determination of the low-mass end IMF in the LMC requires an explicit identification of PMS stars. A preliminary analysis, done for the whole field as a single entity, shows that the IMF slope for the young population present over the entire region is steeper than $\Gamma \simeq-1.7$.

Subject headings: galaxies: individual (Large Magellanic Cloud) - Magellanic Clouds stars: early-type — stars: evolution — stars: pre-main-sequence supernovae: individual (SN 1987A)
\end{abstract}

\section{INTRODUCTION}

SN 1987A has been one of the first targets ever observed with the Hubble Space Telescope (HST). It was first imaged with the FOC on 1990 August 23-24, immediately revealing the high potential of $H S T$ observations, despite the unfortunate problem of spherical aberration, with an impressive display of the supernova itself, flanked by its companion stars, stars 2 and 3, and the famous inner circumstellar ring (Jakobsen et al. 1991; Panagia et al. 1991). The full HST capabilities were reached only after the first refurbishment mission in 1993 December. And surely enough, SN 1987A caught everybody's eye with its beautiful triple ring circumstellar nebula, which was imaged with the new WFPC2 (Burrows et al. 1995; Panagia et al. 1996). Since then, SN

\footnotetext{
${ }^{1}$ Based on observations with the NASA/ESA Hubble Space Telescope, obtained at the Space Telescope Science Institute, which is operated by AURA, Inc., under NASA contract NAS 5-26555.

${ }^{2} \mathrm{On}$ assignment from the Astrophysics Division, Space Science Department of the European Space Agency.
}

1987A has regularly been imaged in a number of broadband and narrowband filters at least once a year as part of the Supernova Intensive Study (SINS; the principal investigator is R. P. Kirshner) project to monitor the evolution of the supernova as well as the changes in the emission of the rings. The combination of these images, always centered on the supernova but taken with different roll angles, resulted in an excellent coverage of an area of about $130^{\prime \prime}$ radius, i.e., about $30 \mathrm{pc}$, around SN 1987A.

SN 1987A is located about $20^{\prime}$ southwest of the edge of the 30 Doradus nebula. This area includes regions of very active star formation, in which different groups of early-type stars are interspersed with $\mathrm{H}$ II regions and supernova remnant (SNR) shells. The association closest to SN 1987A is LH 90, which is located about $5^{\prime}$ to the northeast of the supernova (Lucke \& Hodge 1970) and whose age is much younger than that of the SN 1987A progenitor, i.e., about 4 Myr as compared with the 10-11 Myr as estimated for Sk -69 202 (e.g., Van Dyk, Hamuy, \& Mateo 2000). The supernova itself is surrounded by a number of bright blue 
stars that seem to cluster around it. It is clear that the study of SN 1987A neighborhood offers a unique opportunity to place the supernova explosion in the proper context of stellar evolution and the evolution of stellar populations.

Here, we present the first results of a systematic study of the stellar populations contained in a field of 130" (about 30 pc) radius around SN 1987A, focusing mostly on the properties of the younger populations. We show that several distinct generations of young stars can be identified in addition to the one that gave birth to the $\mathrm{SN}$ progenitor, with ages from $1 \mathrm{Myr}$ or less to at least 100-150 Myr. Thanks to $\mathrm{H} \alpha$ narrowband photometry, we have identified almost 500 strong-line $\mathrm{T}$ Tauri stars: this constitutes the first positive (spectroscopic) detection of moderate-/lowmass (about 1-2 $M_{\odot}$ ) pre-main-sequence (PMS) stars outside the Milky Way. ${ }^{3}$ Their detection provides an excellent tracing of the low mass PMS star spatial distribution, which is compared with that of coeval, more massive stars. On this basis, we show that within the stellar generation of SN 1987A the more massive stars $\left(M>6 M_{\odot}\right)$ are mostly grouped around the $\mathrm{SN}$ progenitor, whereas lower mass stars $\left(1 M_{\odot}<M<2 M_{\odot}\right)$ are more evenly distributed, suggesting that different formation mechanisms are operating for stars of different masses. Finally, we consider and discuss the problem of determining the initial mass function (IMF), concluding that an explicit identification of PMS stars based on spectroscopic criteria is absolutely necessary to separate young, low-mass stars from older population, field stars properly.

\section{OBSERVATIONS AND DATA REDUCTION}

Since 1994, Supernova 1987A has been imaged with the WFPC2 every year as a part of the SINS project. The log of the observations we have used is reported in Table 1 . We did not use the observations of 1995 March 3, since only four bands were taken and they did not provide any significant addition to the portion of sky covered by the others. Among all SINS images taken until 1997, only for one epoch (1997 July) was the field observed in the $\mathrm{H} \alpha$ filter.

\footnotetext{
${ }^{3}$ Existing studies of other young regions (e.g., Gilmozzi et al. 1994; Hunter et al. 1995) in the LMC have already found PMS stars but only in a statistical sense, i.e., identifying them only on the basis of their location in the $\mathrm{H}-\mathrm{R}$ diagram.
}

Since, as shown in $\S \S 4$ and $5, \mathrm{H} \alpha$ images are essential in studying the young population, we complemented the SINS data with the $\mathrm{H} \alpha$ images taken for $H S T$ program 5203 (principal investigator J. Trauger) on 1994 February 3, which overlap almost exactly with the SINS images obtained in 1996 February with broadband filters.

The observations were processed through the standard postobservation data processing system (PODPS) pipeline for bias removal and flat fielding. In all the cases the available images for each filter were combined to remove cosmicray events.

The plate scale of the camera is 0.045 and 0.099 pixel $^{-1}$ in the PC and in each of the three WF chips, respectively. We performed aperture photometry following the prescriptions by Gilmozzi (1990) as refined by Romaniello (1998), i.e., measuring the flux in a circular aperture of 2 pixels radius and the sky background value in an annulus of internal radius 3 pixels and width 2 pixels. Aperture photometry is perfectly adequate for our study because crowding is never a problem in our images. Actually, the average separation of stars from each other is about 1"3 (i.e., 29 pixels in the PC chip and $\sim 13$ pixels in the WF one), which is much larger than the WFPC2 point-spread function (PSF) width. The flux calibration is obtained using the internal calibration of the WFPC2 (Whitmore 1995), which is typically accurate to within $\pm 5 \%$. We use the spectrum of Vega as photometric zero point. However, since we have used the IRAF synphot synthetic photometry package to compute the theoretical data numbers to be compared to the observed ones, the choice of the zero points has no influence on the final results.

The brightest stars in each CCD chip are saturated (i.e., most stars brighter than 17.5 in the F555W band). We recover their photometry either by fitting the unsaturated wings of the PSF for moderately saturated stars, i.e., with no saturation outside the central 2 pixel radius, or by following the method developed by Gilliland (1994) for heavily saturated stars. In most cases, the achieved photometric accuracy is better than 0.05 mag. Full description of the methods used can be found in Romaniello (1998) and Romaniello et al. (2000). As a sanity check we have compared our photometry in the $B$ and $V$ bands with the one obtained with ground based observations (28 stars brighter than $V \simeq 20$ within $30^{\prime \prime}$ from SN 1987A; Walker \& Suntzeff

TABLE 1

Log of ObSERvations Centered on SN 1987A

\begin{tabular}{|c|c|c|c|c|}
\hline \multirow[b]{2}{*}{ Filter NAME } & \multicolumn{3}{|c|}{ EXPOSURE TIME (s) } & \multirow[b]{2}{*}{ Comments } \\
\hline & 1994 September a & 1996 February $^{\mathrm{b}}$ & 1997 July $^{\mathrm{c}}$ & \\
\hline F255W ...... & $2 \times 900$ & $1100+1400$ & $2 \times 1300$ & UV Filter \\
\hline F336W ...... & $2 \times 600$ & $2 \times 600$ & $2 \times 800$ & $U$ filter \\
\hline F439W ...... & $2 \times 400$ & $350+600$ & $2 \times 400$ & $B$ filter \\
\hline F555W ..... & $2 \times 300$ & $2 \times 300$ & $2 \times 300$ & $V$ filter \\
\hline F675W ...... & $2 \times 300$ & $2 \times 300$ & $2 \times 300$ & $R$ filter \\
\hline F814W ...... & $2 \times 300$ & $2 \times 300$ & $2 \times 400$ & $I$ filter \\
\hline F502N ........ & $4 \times 1200$ & $1100+1500$ & $2 \times 1300+4 \times 1400$ & {$[\mathrm{O}$ III $] \lambda 5007 \AA$} \\
\hline F656N ............ & $\ldots$ & $1100+1300^{d}$ & $4 \times 1400$ & $\mathrm{H} \alpha$ \\
\hline F658N ........ & $4 \times 1200$ & $1100+1500$ & $\ldots$ & {$[\mathrm{N} \mathrm{III}] \lambda 6584 \AA^{\mathrm{e}}$} \\
\hline
\end{tabular}

a 1994 September 24, proposal 5753.

b 1996 February 6, proposal 6020.

c 1997 July 10, except for F502N taken on 1997 July 12, proposal 6437.

d Not from SINS (principal investigator is John Trauger), taken on 1994 February 3.

${ }^{\mathrm{e}}$ With $\sim 25 \% \mathrm{H} \alpha$ contamination. 
1990) and found an overall excellent agreement, i.e., rms deviations less than 0.05 mag in both bands, except for the obvious cases of few stars that appear as pointlike objects in ground based images but are resolved by HST observations.

Figure 1 shows the entire field as observed in a mosaic of $B, V$, and $R$ broadband plus the [O $\mathrm{III}]$ and $\mathrm{H} \alpha$ narrowband images. As mentioned in the introduction, SN 1987A appears to be part of a group of early-type stars whose spatial density is significantly higher in the neighborhood of the supernova than in the remaining area, suggesting the presence of a physical group and/or a small cluster. Quantitatively, the number of stars brighter than $B=18 \mathrm{mag}$ in the inner $20^{\prime \prime}$ radius region (15 stars) is about $23 \%$ of the total 64 such stars contained within $130^{\prime \prime}$ radius field, although the former has an area of only $4 \%$ of the total field.

We identify the stars in the F555W exposure and then measure their magnitudes in all of the other filters. The total number of stars detected in this way is 21,955 . For about 12,340 of them the photometric accuracy is better than 0.1 mag in the $V, R$, and $I$ filters. The number of stars with accuracy better than 0.1 mag drops to 6825 in the $B$ band, and only 786 stars have a UV filter uncertainty smaller than $0.2 \mathrm{mag}$.

\section{COLOR-MAGNITUDE DIAGRAMS AND H-R DIAGRAMS}

Figure 2 displays the color-magnitude diagrams (CMDs) for four combinations of bands. In order to select stars with overall good photometry, we have used the average error in five bands $\left(\bar{\delta}_{5}\right)$, excluding the UV:

$$
\bar{\delta}_{5}=\sqrt{\frac{\delta_{\mathrm{F} 336 \mathrm{~W}}^{2}+\delta_{\mathrm{F} 439 \mathrm{~W}}^{2}+\delta_{\mathrm{F} 555 \mathrm{~W}}^{2}+\delta_{\mathrm{F} 675 \mathrm{~W}}^{2}+\delta_{\mathrm{F} 814 \mathrm{~W}}^{2}}{5}}
$$

The black dots in each CMD in Figure 2 are the 6695 stars with $\bar{\delta}_{5}<0.1 \mathrm{mag}$. The error threshold of $\bar{\delta}_{5}<0.1$ reflects itself as a magnitude threshold at $m_{\mathrm{F} 555 \mathrm{~W}} \simeq 23$. We estimate that completeness at this magnitude limit is very close to $100 \%$ because the density of detected stars down to 23rd magnitude is rather low, i.e., about one star per 4.6

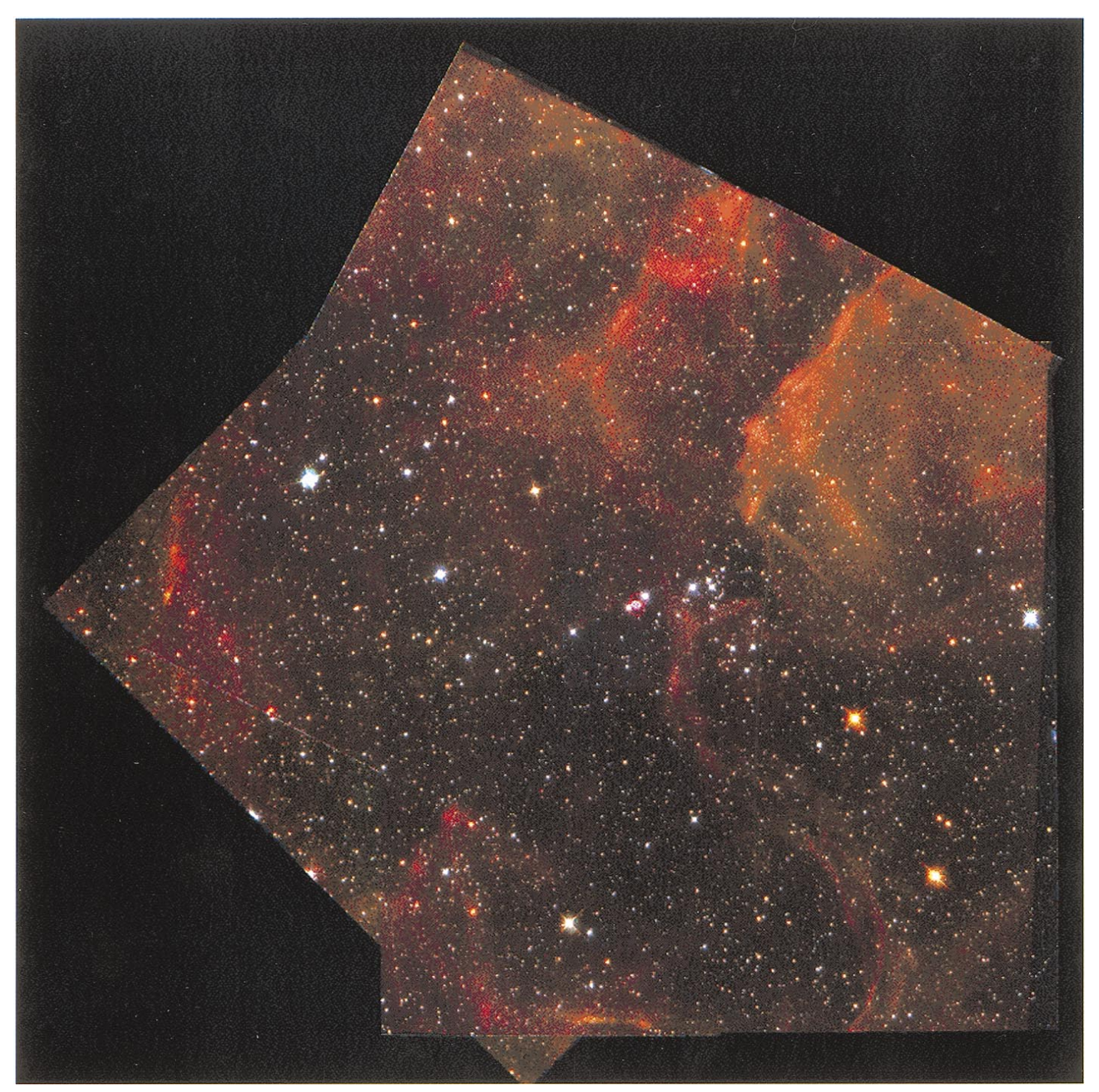

FIG. 1.-Field centered on SN 1987A (about 130" radius) as observed in the combination of the $B, V$, and $I$ broadbands plus the [O III] and $\mathrm{H} \alpha$ narrowband images. The blue, bright star about $94^{\prime \prime}$ northeast of SN $1987 \mathrm{~A}$ is the brightest $\left[\log \left(L / L_{\odot}\right) \simeq 5.8\right]$ and most massive $\left(\sim 60 M_{\odot}\right)$ star in the field (see $\S \S 4$ and 5). 

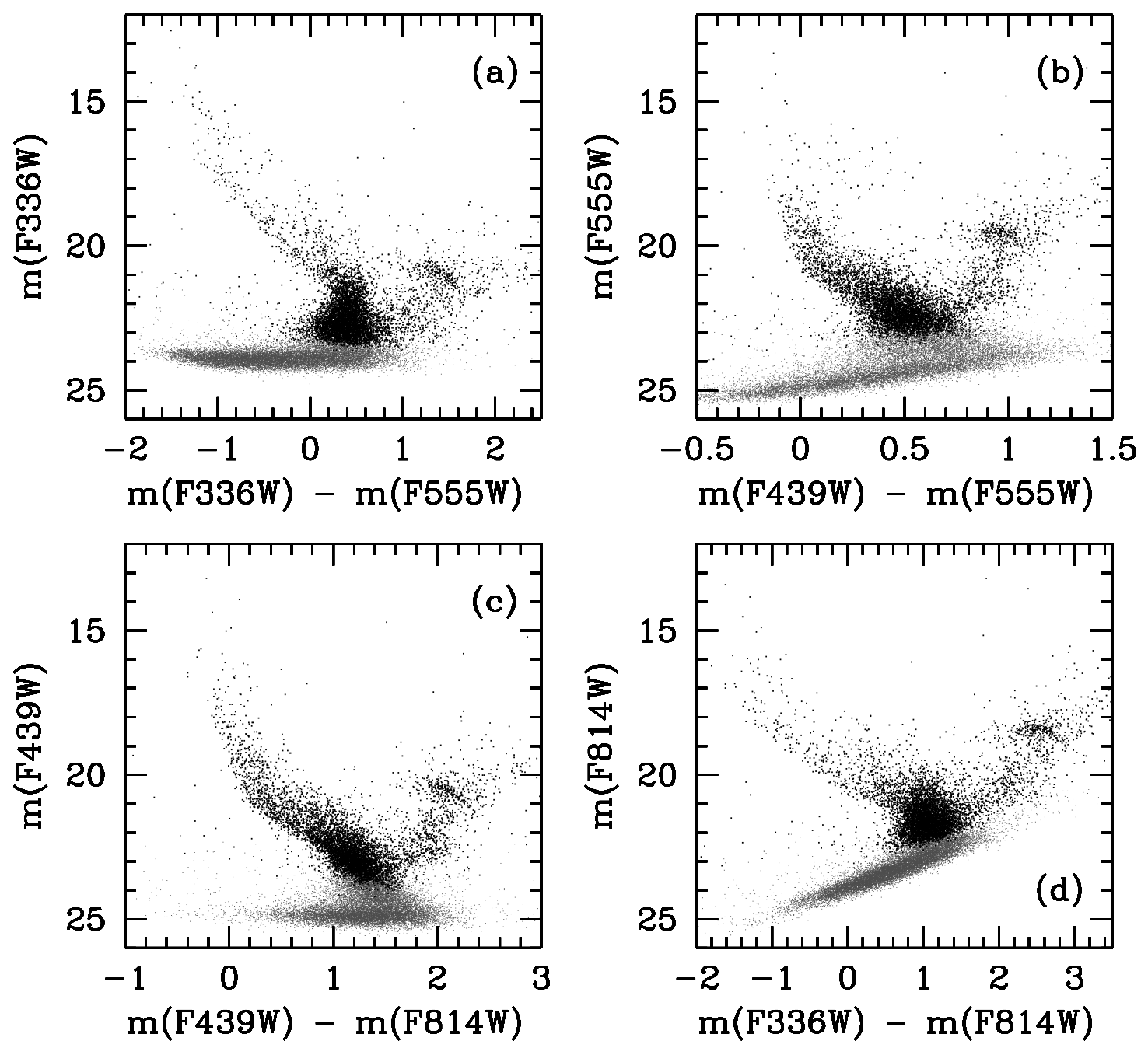

FIG. 2.-Color-magnitude diagrams for four combination of filters. The gray dots are stars with average errors (as defined in eq. [1]) $\bar{\delta}_{5}>0.1$, whereas the black dots are the 6695 stars with $\bar{\delta}_{5}<0.1$.

$\operatorname{arcsec}^{2}$ area. Thus, conservatively adopting an effective PSF area of $0.28 \operatorname{arcsec}^{2}$ (i.e., a $3 \mathrm{WF}$ pixel radius circular area) and defining $f$ as the probability of having one star brighter than 23rd magnitude falling in any one PSF area $(f=0.28 / 4.6=0.061)$, we find that a simple application of Poisson statistics predicts that only a fraction of $\left[1-f e^{-f} /\right.$ $\left.\left(1-e^{-f}\right)\right]=0.031$ of the stars may overlap with other stars.

It is apparent that, despite the high quality of the measurements (internal uncertainties less than $0.1 \mathrm{mag}$ ), the various features of the CMDs, such as the zero-age main sequence (ZAMS) and the sequence of binaries for the earlytype stars and the red giant clump for the more evolved populations, are rather "fuzzy" and not sharply defined. Although this is due in part to the presence of several stellar populations projected on each other (see $\$ \S 4,5$, and 6), most of the problem arises from the fact that reddening is not quite uniform over the field, thus causing the points of otherwise identical stars to fall in appreciably different locations of the CMDs.

The large number of bands available (six broadband filters), covering a wide baseline (more than a factor of 4 in wavelength, extending from $\sim 2300$ to $\sim 9600 \AA$ ), provide us with a sort of wideband spectroscopy that defines the continuum spectral emission distribution of each star quite well. Therefore, by comparison with model atmospheres (Bessel, Castelli, \& Plez 1998), one can fit the six band observations of each stars and solve for three unknowns simultaneously, namely, the effective temperature, $T_{\text {eff }}$, the reddening, $E(B-V)$, and the angular radius, $R / D$. As is well known, the solution in the plane $T_{\text {eff }}-E(B-V)$ may not be unique for stars with effective temperatures lower than about $9000 \mathrm{~K}$. Therefore, we first solve for the full set of parameters, $T_{\text {eff }}, E(B-V)$, and $R / D$, only for stars suitably selected on the basis of reddening-free colors. These turn out to have temperature either higher than $10,000 \mathrm{~K}$ or between 6750 and $8500 \mathrm{~K}$. For each of the remaining stars, we adopt the average reddening of its neighbors and solve for only two parameters, $T_{\text {eff }}$, and $R / D$. Finally the stellar luminosity is computed from the derived $T_{\text {eff }}$ and $R / D$ values, adopting a distance to SN $1987 \mathrm{~A}$ of $51.4 \mathrm{kpc}$ (Panagia 1999; Panagia et al. 2000, in preparation; Romaniello et al. 2000). A full account of this analysis will be presented in a forthcoming paper (Romaniello et al. 2000, in preparation; see also Romaniello 1998). Of the total sample of 21,995 stars, the uncertainty in the effective temperature is lower than $12 \%$ for 9474 stars, most of which with $\log \left(L / L_{\odot}\right)>0$. Also, we were able to obtain individual reddening determinations for 2510 stars (i.e., about $11 \%$ of 


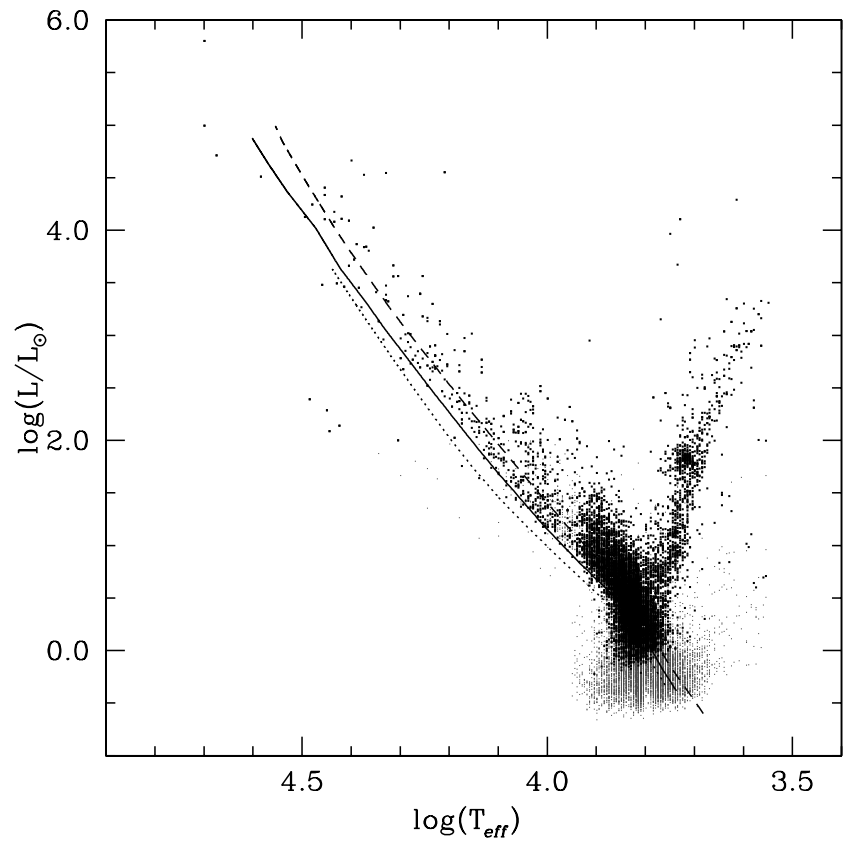

FIG. $3 a$

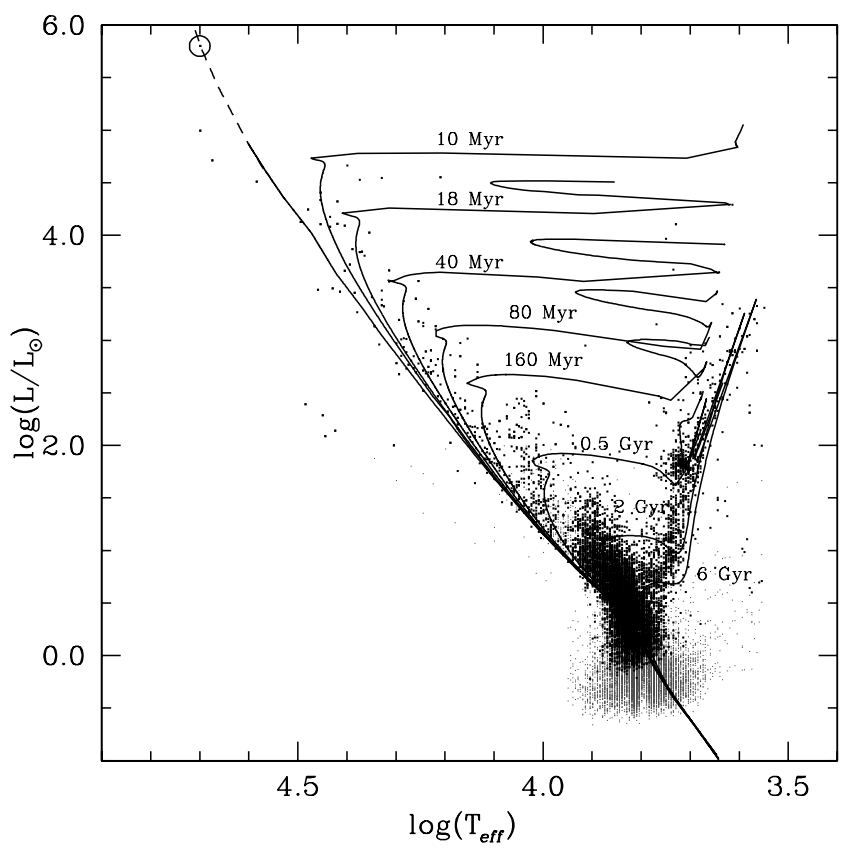

FIG. $3 b$

FIG. 3.-H-R diagram for the stars in the WFPC2 field. The gray dots are stars with average errors (as defined in eq. [1]) $\bar{\delta}_{5}>0.1$, whereas the black dots are the 6695 stars with $\bar{\delta}_{5}<0.1$. (a) Comparison with the theoretical ZAMS for different $Z$ values: $Z=Z$ (long-dashed line), $Z=0.3 Z$ (solid line), and $Z=Z_{\odot} / 20$ (dotted line). (b) Comparison with a number of post-MS isochrones (Brocato \& Castellani 1993; Cassisi et al. 1994). The brightest star in the field, possibly a $\sim 60 M_{\odot}$ star younger than $1 \mathrm{Myr}$, is marked with a circle at the top of the diagram.

the whole sample). On average, this corresponds to a reddening value every $13 \operatorname{arcsec}^{2}$, which implies that the extinction distribution over the entire field was mapped down to a scale size of about 3."5 (i.e., about $1 \mathrm{pc}$ ).

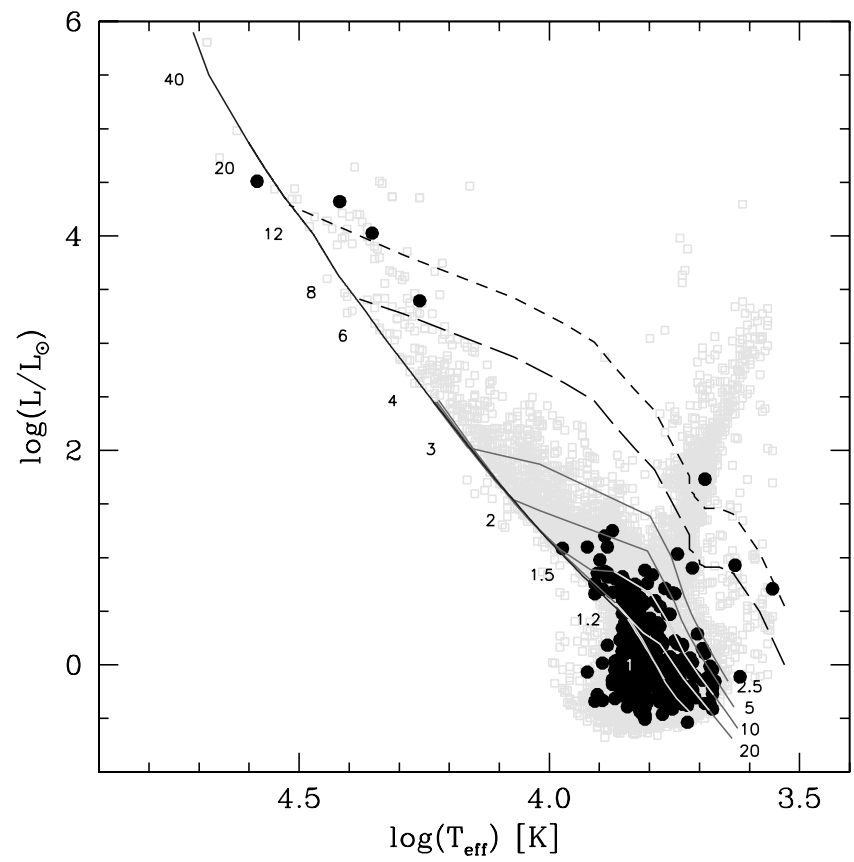

FIG. 4.-H-R diagram displaying the positions of the stars with strong $\mathrm{H} \alpha$ excess (dots) overlaid on the general stellar population (gray squares) found in the WFPC2 field. For reference, we show the theoretical ZAMS (Brocato \& Castellani 1993) with marked position for stars of various masses. Also shown are 2.5-20 Myr PMS isochrones (Siess et al. 1997) and the birthlines for accretion rates of $10^{-4}$ (short-dashed line) and $10^{-5} \mathrm{M}_{\odot}$ $\mathrm{yr}^{-1}$ (long-dashed line; Palla \& Stahler 1993).
The reddening is clearly not constant over the field, having an average value of $\langle E(B-V)\rangle=0.203$ and an rms deviation $\sigma[E(B-V)]=0.072$. The average value is very close to the reddening determined by Scuderi et al. (1996) in the direction of star $2(0.19 \pm 0.02)$ and by Walborn et al. (1993) from an analysis of photometric ground based observations of early-type stars in the same field. The reddening spatial distribution does not correlate well with the distribution of nebular emission. Moreover, the reddening fluctuations do not show any obvious pattern, suggesting the presence of a highly clumped diffuse medium (cf. Romaniello 1998; Romaniello et al. 2000, in preparation).

\section{STELLAR POPULATIONS AND AGES}

The resulting H-R diagram $\left[\log \left(L / L_{\odot}\right)\right.$ vs. $\log T_{\text {eff }}$ plot $]$ is shown in Figure 3. Its inspection confirms the early findings of Walker \& Suntzeff (1990) and Walborn et al. (1993) and reveals that the distribution of stars in the H-R diagram is clearly bounded toward high temperatures, identifying a ZAMS that corresponds to a metallicity $Z \simeq Z_{\odot} / 3$ (see Fig. $3 a$ ). This value agrees well with the general abundances measured in the LMC as well as the more local estimates derived from spectroscopy of the circumstellar rings (Panagia et al. 1996) and star 2 (Scuderi et al. 1996). Therefore, in the following the observed points will be compared with $Z=0.006$ isochrones, either from Brocato \& Castellani (1993) and Cassisi, Castellani, \& Straniero (1994) for post-MS evolution or from Siess, Forestini, \& Dougados (1997) for PMS evolution.

With the exception of one very bright $\operatorname{star}^{4}$ (readily recognizable in Figure 1 and denoted with a larger symbol in

\footnotetext{
${ }^{4}$ This star has $\log \left(T_{\text {eff }}\right)=4.7, \log \left(L / L_{\odot}\right)=5.8$, which corresponds to a mass of $\sim 60 M_{\odot}$ and suggests an age probably younger than $10^{6} \mathrm{yr}$.
} 
Figs. $3 b$ and $6 c$ ), the positions of the most luminous blue stars (see Fig. 3b) fall on isochrones corresponding to ages around 10-14 Myr, which make them coeval to SN 1987A's progenitor and star 2 (cf. Scuderi et al. 1996, and references therein). There are a number of stars at intermediate luminosities and temperatures that indicate ages up to $100-150$ Myr and initial masses down to $4 M_{\odot}$. This is especially clear from the position of the yellow/red supergiants around $\log T_{\text {eff }} \sim 3.6-3.8$ and $\log \left(L / L_{\odot}\right) \sim 2-3$.

The lower MS and the red giants are mostly old population stars, consistent with a metallicity either identical to, or slightly lower than, that of the young components. No single age can explain the distribution of the old population, and substantial star formation between $600 \mathrm{Myr}$ and $6 \mathrm{Gyr}$ is required to account for the observations (cf. Fig. 3b).

In summary, we find evidence for a series of star formation episodes spanning from several billion years ago until at least 1-2 Myr and, possibly, still ongoing (cf. Fig. 4 and $\S 5$ ). We identify a generation of stars with ages about 12 Myr, which makes them coeval to the supernova progenitor and may include up to $35 \%$ of the young population in this field (defined as stars with ages less than $100 \mathrm{Myr}$ ). The old field population requires continued star formation over a broad interval of $0.6-6$ Gyr. From all of these results, it is clear that, since even the young stars belong to a mixture of populations of different ages, a study of the IMF is very hard and requires a proper separation of the various stellar generations to avoid systematic biases and errors.

\section{PRE-MAIN-SEQUENCE STARS AND THE YOUNG POPULATION}

By comparing the magnitudes in the $R$ band (F675W) with those measured with the narrowband $\mathrm{H} \alpha$ filter (F656N), we can identify stars with sufficiently strong $\mathrm{H} \alpha$ emission. Considering the throughputs of the F675W and F656N filters (cf. Biretta 1996), we estimate that an $\mathrm{H} \alpha$ emission line with equivalent width of $8 \AA$ will produce a color excess $m(R)-m(\mathrm{H} \alpha) \simeq 0.3 \mathrm{mag}$. Therefore, we define as stars with strong $\mathrm{H} \alpha$ excess the ones with color excess $m(R)-m(\mathrm{H} \alpha)$ that is both greater than 0.3 and greater than 4 times the photometric uncertainty, i.e., $4\left[\sigma^{2}(R)+\sigma^{2}(\mathrm{H} \alpha)\right]^{1 / 2}$. The requirement on the error assures that a measured excess is highly significant, and the high equivalent width cutoff excludes any contamination by normal stars with chromospheric activity because they have equivalent widths of less than $3 \AA$ (e.g., Frasca \& Catalano 1994). The positions in the $\mathrm{H}-\mathrm{R}$ of $\mathrm{H} \alpha$ excess stars are shown in Figure 4 and compared with the distribution of all other stars.

We identify the luminous and bright ones (four stars), which are near the MS, as Be stars. One of them is star 3, one of the two companions to SN 1987A (see Walborn et al. 1993, and references therein); its high variability qualifies it for a "typical" Be star (cf. Jaschek et al. 1980). However, its emission lines are too narrow (cf. Walborn et al. 1993) to conform to the characteristics of canonical Be stars. A possible explanation is that star 3 is seen almost pole-on. On the other hand, we cannot exclude the possibility that star 3 belongs to the same class of HAeBe stars, i.e., massive PMS stars (Herbig 1960), as the six possible candidates found near the center of the LMC bar by Beaulieu et al. (1996) and discussed by Lamers, Beaulieu, \& De Wit (1999). They concluded that the location in the H-R diagram of their candidate $\mathrm{HAeBe}$ stars indicates a prevailing accretion rate of about $10^{-4} M_{\odot} \mathrm{yr}^{-1}$, i.e., 10 times higher than the "canonical value" of $10^{-5} M_{\odot} \mathrm{yr}^{-1}$ as appropriate for the Milky Way (cf. Palla \& Stahler 1993). Also,our four Be stars, if they are considered to be stars evolving toward the main sequence, would require a considerably brighter birthline for the LMC than is found for Milky Way stars (see Fig. 4), again indicating accretion rates up to $10^{-4} M_{\odot} \mathrm{yr}^{-1}$. If the PMS nature of such stars is confirmed by detailed spectroscopic studies, these results would imply that star formation in a low-metallicity environment proceeds at a substantially different pace than it does in our Galaxy, at least for massive stars.

We identify the redder and fainter stars with strong $\mathrm{H} \alpha$ excess (488 stars, 275 of which have very accurate temperature determinations, $\delta \log T_{\text {eff }}<0.05$ ) as strong-line T Tauri stars, i.e., PMS stars with circumstellar material, remnants of their protostellar cocoons. ${ }^{5}$ Their $\mathrm{H} \alpha$ excesses correspond to $\mathrm{H} \alpha$ equivalent widths that range between 8 and $360 \AA$, with $90 \%$ of the $W_{\text {eq }}(\mathrm{H} \alpha)$ values falling within 11 and $85 \AA$. These properties are very similar to those of Galactic strong-line T Tau stars (e.g., Herbig \& Bell 1988; Fernandez et al. 1995), although we note that the $\mathrm{H} \alpha$ equivalent width distribution of $\mathrm{T}$ Tau stars in our field is more skewed toward lower values those in the Galaxy (cf. Fig. 5). This difference is easily explained as an age effect, in that classical Galactic T Tau stars are much younger (ages below $5 \mathrm{Myr}$ ) than those in our field. Comparing the positions of T Tauri stars in the H-R diagram with PMS evolution model calcu-

\footnotetext{
${ }^{5}$ Confirmation of the reality of the measured $\mathrm{H} \alpha$ excesses and the T Tau identification is provided by both a visual inspection of the images, which excludes the presence of nebular filamentary structures crossing each candidate star image, which may mimic an apparent excess, and measurements of the stellar fluxes in the [O III] and [N II] narrowband filters (F502N and F658N, respectively) that for all of the bona fide T Tau candidate stars give line emission that are appropriately much lower than the emission measured for the $\mathrm{H} \alpha$ line.
}

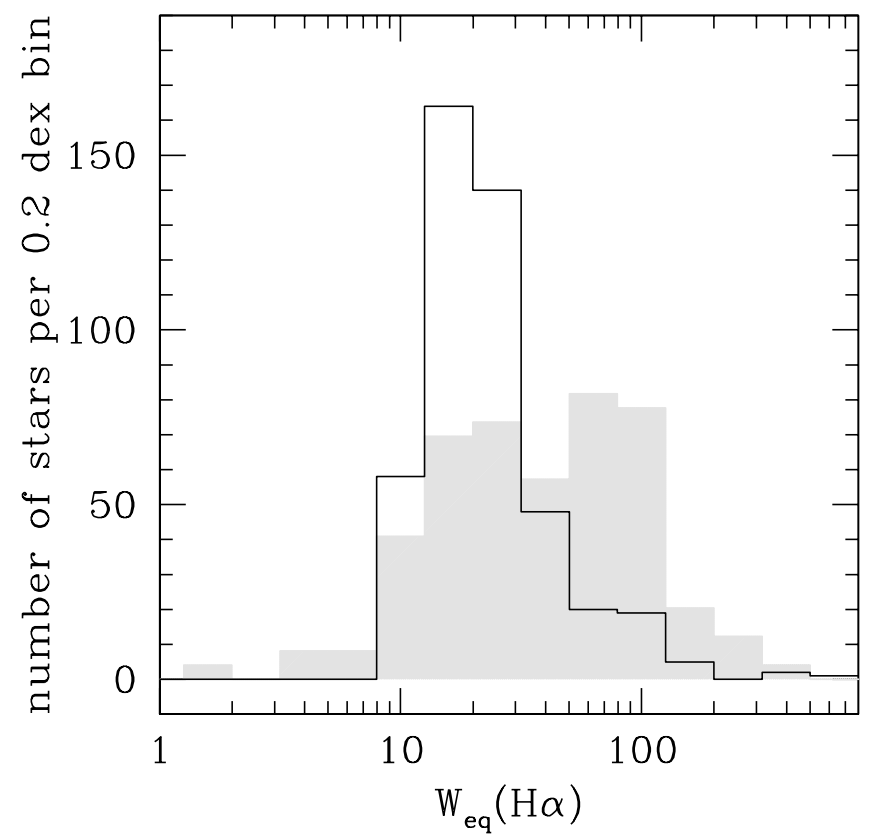

FIG. 5.-Distribution of number of stars with strong $\mathrm{H} \alpha$ emission per unit of 0.2 dex bin of the $\mathrm{H} \alpha$ equivalent width, $W_{\mathrm{eq}}(\mathrm{H} \alpha)$. Candidate Be stars have been excluded from this histogram. The shaded histogram is the corresponding distribution for Galactic strong-line T Tau stars (Fernandez et al. 1995). 


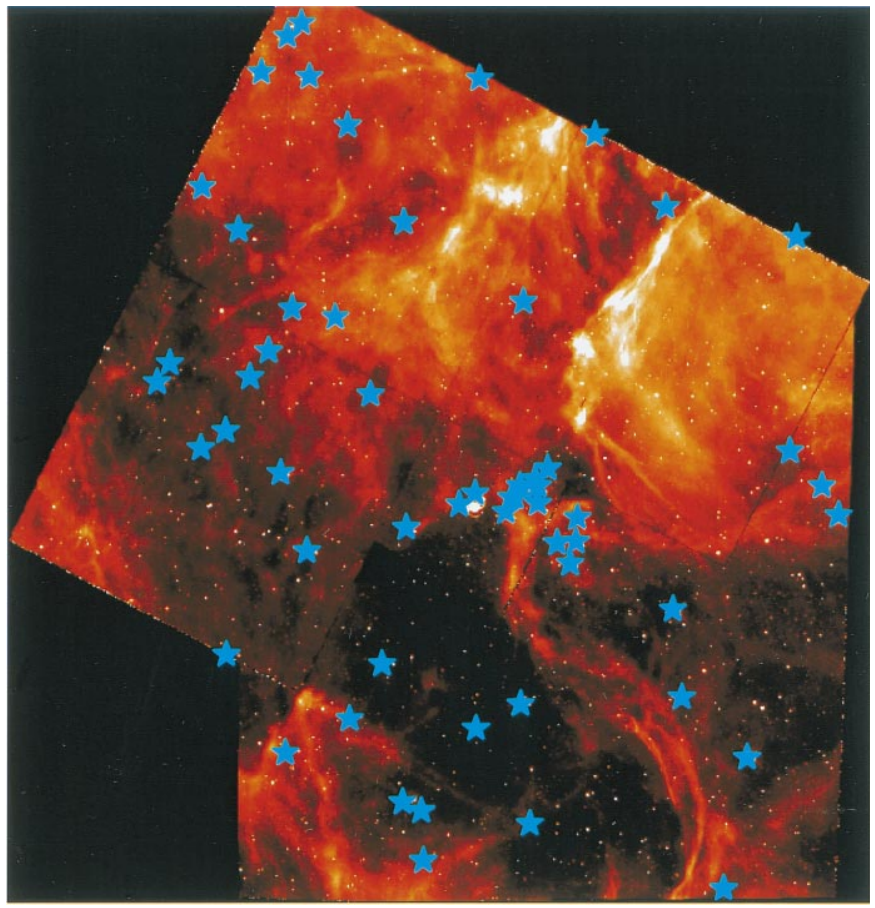

FIG. $6 a$

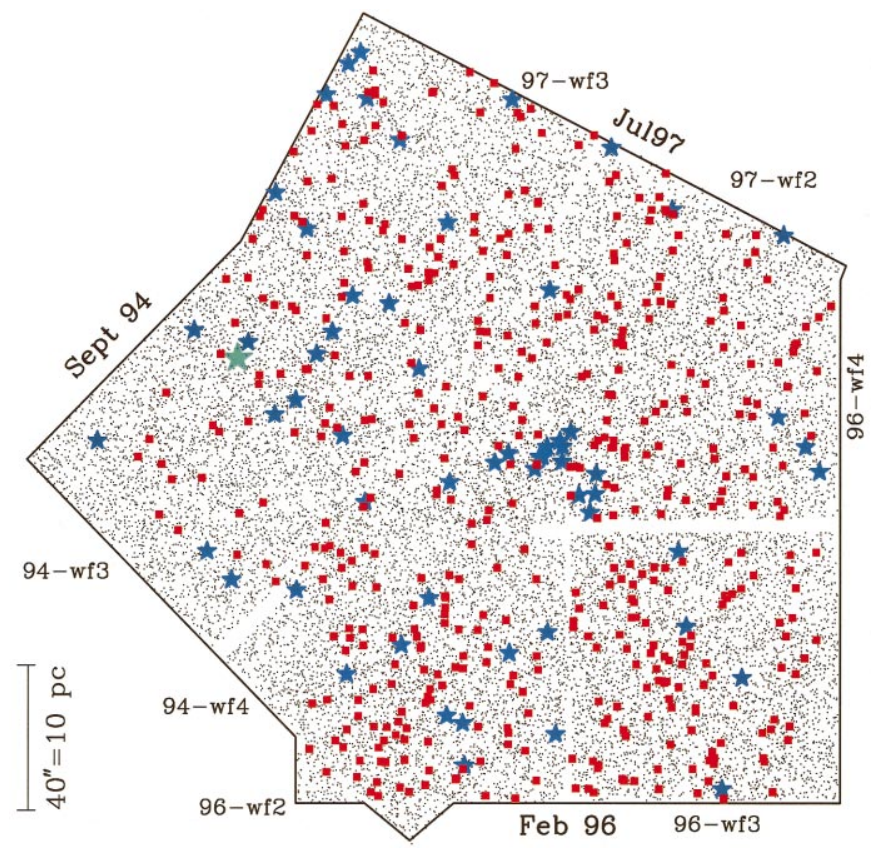

FIG. 6c

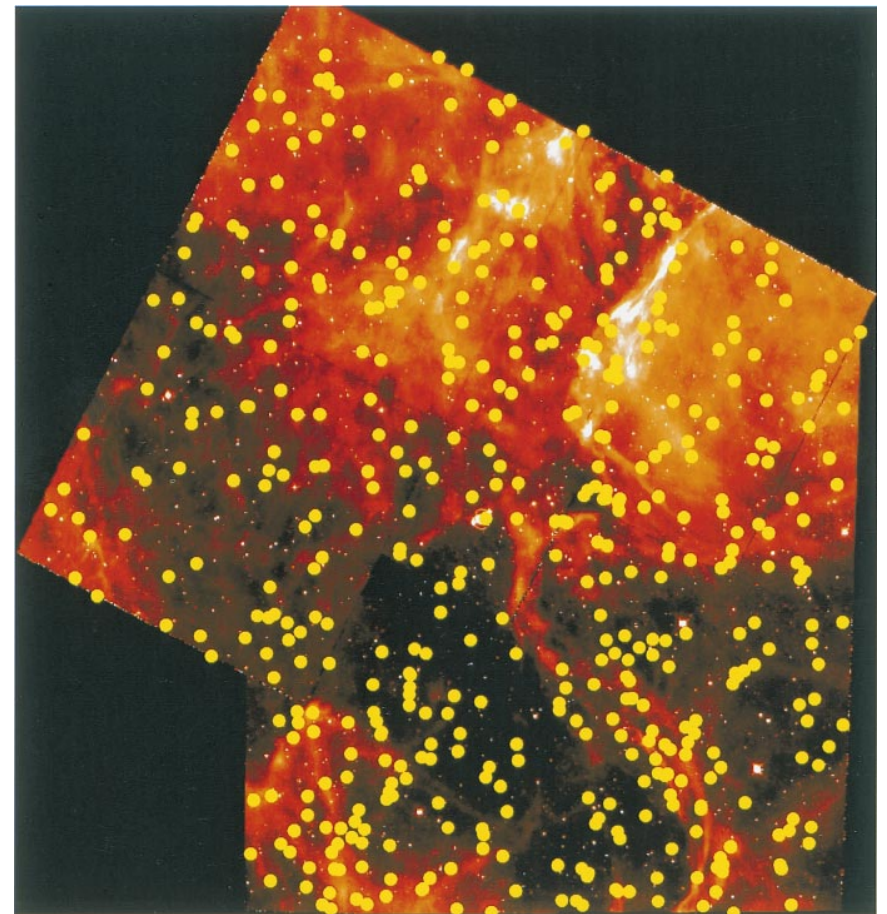

FIG. $6 b$

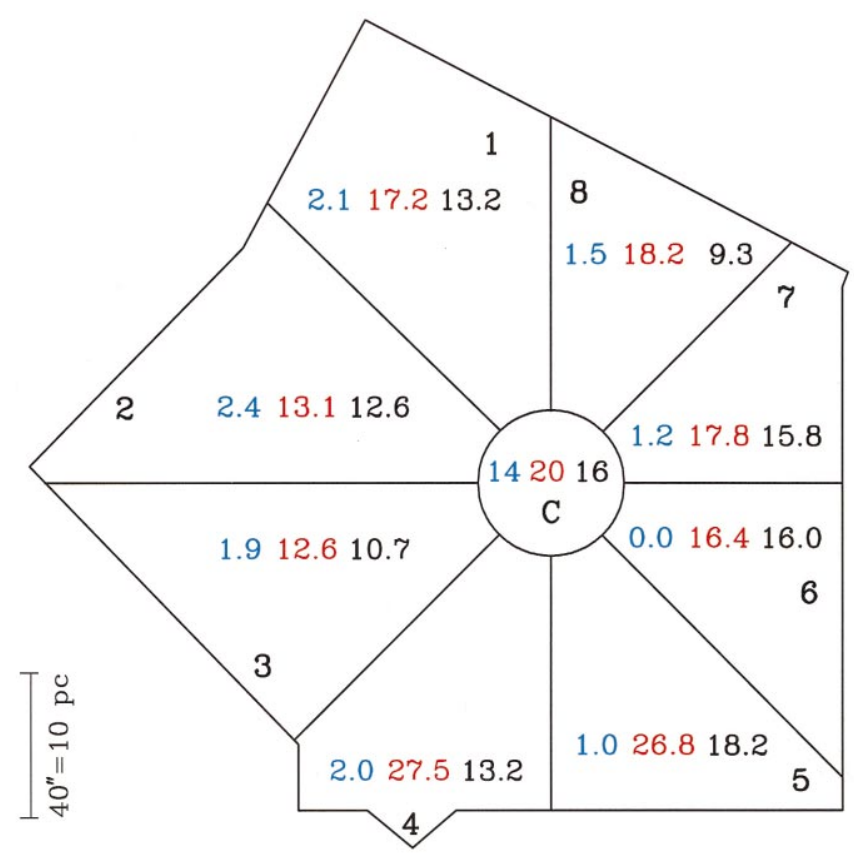

FIG. 6d

Fig. 6. - Comparison of the spatial distributions of massive stars $\left(M>6 M_{\odot}\right.$, five-pointed stars) and PMS stars $\left(M<2 M_{\odot}\right.$, squares) belonging to the same younger population. North is up, and east is to the left. (a) Massive stars overlaid on a mosaic of WFPC2 H $\alpha$ images. (b) PMS stars overlaid on a mosaic of WFPC2 H $\alpha$ images. (c) Massive stars (blue five-pointed stars) and PMS stars (red squares) overlaid on the general field including 21,995 stars (small dots). The brightest star (larger, pale blue five-pointed star) has $\log \left(L / L_{\odot}\right) \simeq 5.8$. (d) Number of massive stars (blue), PMS stars (red) and Red Giant Clump stars (black) in a central circle centered on SN 1987A and in eight sectors (cf. Table 2 and $\S 5$ ).

lations by Siess et al. (1997), we find that most of them have masses in the range $1-2 M_{\odot}$ and ages between $1-2$ and 20 Myr and possibly beyond (see Fig. 4). Note that the presence of T Tau stars on or very close to the birthline even suggests that some star formation is still ongoing in this field. Overall, $T$ Tau stars represent about $4 \%$ of the total number of stars present in the same area of the
H-R diagram [approximately $\log \left(T_{\text {eff }}\right)=3.65-3.95$ and $\left.\log \left(L / L_{\odot}\right)<1\right]$, making them very hard to identify without positive spectroscopic diagnostics.

We would like to stress that this result constitutes the first positive detection of moderate- to low-mass (about 1-2 $\left.M_{\odot}\right)$ PMS stars outside the Milky Way. In fact, although previous studies of other young regions of the LMC (e.g., 
TABLE 2

Spatial Distribution of Stars around SN 1987A

\begin{tabular}{|c|c|c|c|c|c|c|c|}
\hline \multirow[b]{2}{*}{ REGION } & \multirow[b]{2}{*}{ ArEA $^{a}$} & \multicolumn{2}{|c|}{ Massive Stars $^{b}$} & \multicolumn{2}{|c|}{ T TAURI STARs ${ }^{c}$} & \multicolumn{2}{|c|}{ RED GIANTs $^{d}$} \\
\hline & & Number & Density & Number & Density & Number & Density \\
\hline ....... & 1.00 & 14 & $14 \pm 3.7$ & 20 & $20.0 \pm 4.5$ & 16 & $16.0 \pm 4.0$ \\
\hline $1 \ldots \ldots \ldots \ldots$ & 4.70 & 10 & $2.1 \pm 0.7$ & 81 & $17.2 \pm 1.9$ & 62 & $13.2 \pm 1.7$ \\
\hline $2 \ldots \ldots \ldots \ldots$ & 3.80 & 9 & $2.4 \pm 0.8$ & 50 & $13.2 \pm 1.9$ & 48 & $12.6 \pm 1.8$ \\
\hline ........ & 3.16 & 6 & $1.9 \pm 0.8$ & 40 & $12.6 \pm 2.0$ & 34 & $10.7 \pm 1.8$ \\
\hline$\ldots \ldots$ & 3.02 & 6 & $2.0 \pm 0.8$ & 83 & $27.5 \pm 3.0$ & 40 & $13.2 \pm 2.1$ \\
\hline n......... & 3.14 & 3 & $1.0 \pm 0.6$ & 84 & $26.8 \pm 2.9$ & 57 & $18.2 \pm 2.4$ \\
\hline $6 \ldots \ldots \ldots \ldots$ & 2.31 & 0 & $\overline{0}$ & 38 & $16.4 \pm 2.7$ & 37 & $16.0 \pm 2.6$ \\
\hline ........ & 2.41 & 3 & $1.2 \pm 0.7$ & 43 & $17.8 \pm 2.7$ & 38 & $15.8 \pm 2.6$ \\
\hline $8 \ldots \ldots \ldots \ldots$ & 2.69 & 4 & $1.5 \pm 0.7$ & 49 & $18.2 \pm 2.6$ & 25 & $9.3 \pm 1.9$ \\
\hline Total...... & 26.23 & 55 & $2.1 \pm 0.3$ & 488 & $18.6 \pm 0.8$ & 357 & $13.6 \pm 0.7$ \\
\hline
\end{tabular}

NoTE.- Observed numbers and derived densities of the various types of stars in the regions illustrated in Fig. $4 d$. The quoted errors are the expected fluctuations according to Poisson statistics.

${ }^{\text {a }}$ In units of the area of the central cluster $\left(20^{\prime \prime}\right.$ radius, i.e., $\left.1207 \operatorname{arcsec}^{2}\right)$.

b $\log \left(L / L_{\odot}\right)>3, M>6 M_{\odot}$.

c $\mathrm{EW}(\mathrm{H} \alpha) \gtrsim 8 \AA, 1 M_{\odot}<M<2 M_{\odot}$.

d $3.65<\log T_{\text {eff }}<3.9$ and $1.5<\log \left(L / L_{\odot}\right)<2.1$, LMC field population.

NGC 1850 by Gilmozzi et al. 1994; R136 cluster by Hunter et al. 1995, etc.) have already identified low-mass "PMS stars," those identifications were entirely circumstantial in that they were based exclusively on the location of the candidate stars in the H-R diagram. In the following we will show that, because of heavy contamination by older, field populations of low mass stars, such an identification method may, at best, have a merely statistical value and may lead to an overestimate of the actual number of lowmass stars in a star-forming region.

In Figures $6 a$ and $6 b$ we display the spatial distribution of massive stars, i.e., stars with $M>6 M_{\odot}$ and strong T Tauri stars as defined above, overlaid on a mosaic of the $\mathrm{H} \alpha$ images. We see that massive stars are strongly concentrated near SN 1987A (14 of 55 are within 20" of the supernova), and the remaining ones are mostly located East of SN 1987A (31 out of 41). The PMS stars, on the other hand, do not show any strong spatial concentration, although one can notice that the number density of PMS stars on the northeast side of SN 1987A is appreciably lower than on the southwest side. No obvious correlation is seen between either class of stars and the ionized gas features: this may indicate that the region is rapidly losing memory of the star formation process once the most massive stars, say, those with $M>20 M_{\odot}$, have evolved and died, producing SN II explosions.

From Figure $6 c$, which displays the distributions of the two classes of stars in one and the same plot, it is apparent that they are distributed in substantially different manners. To better quantify this statement, we have divided the field into nine regions: a circle of $20^{\prime \prime}$ radius around SN 1987A and eight radial sectors, as shown in Figure $6 d$.

Table 2 summarizes the areas of the nine regions (note that for each sector the area reported in Table 2 corresponds to the area for which both broadband and $\mathrm{H} \alpha$ observations are available), their star number counts, and the corresponding star densities per unit area. An inspection of this table reveals that there are enormous variations in the proportion of massive stars relative to low-mass stars: in particular, the numbers of massive and low-mass stars in the central area are almost the same, while in other areas the low-mass stars are 7-28 times more numerous than massive stars. These differences are highly significant because they greatly exceed the expected Poissonian fluctuations, also given in Table 2. An experimental check of this fact is provided by similar statistics on the number of stars belonging to the so-called Red Giant Clump, i.e., stars of a much older population, which, therefore, is expected to be uniformly distributed over the field. Indeed, as seen in Table 2, the observed number densities for Red Giant Clump stars show fluctuations that are perfectly compatible with Poisson statistics.

Another point worth noting is that the average density of T Tau stars in sectors 2 and 3 (i.e., east of SN 1987A) is $12.9 \pm 1.4$, i.e., $3.5 \sigma$ lower than the average $18.6 \pm 0.8$, whereas in sectors 4 and 5 (south of SN 1987A) it is $3.9 \sigma$ higher $(27.4 \pm 2.1)$. It would be tempting to interpret such a systematic difference in terms of a star formation "wave" or "front" that sweeps the region. On the other hand, we do not find any significant difference in ages among PMS stars in the various sectors, suggesting that, rather than dealing with some sort of propagating star formation, the key factor here is the overall efficiency of the star formation process that varies from place to place. Also, we note that there is no enhancement in the density of T Tau stars around the SN 1987A cluster nor near the brightest star in the observed field (see Fig. 6c), lending support to the idea that the process of formation of low-mass stars may be distinct from the one leading to the formation of massive stars.

\section{SN 1987A STELLAR GROUP: A "TYPICAL" CLUSTER?}

In $\S 4$ we have argued that massive stars are strongly concentrated around SN 1987A. This is clearly seen in Figure $7 a$ (see also Fig. 1), which shows an area of about $50^{\prime \prime} \times 50^{\prime \prime}$ around SN 1987A: 14 stars brighter than $10^{3} L / L_{\odot}$ are found in the vicinities of the supernova. Actually, SN 1987A and its companions, stars 2 and 3, are at the eastern edge of the stellar group, whose center almost coincides with a compact "core" of eight blue stars. We note that the size of this stellar group associated with SN 1987A (about $9 \mathrm{pc}$ ) is similar to the average size of stellar 


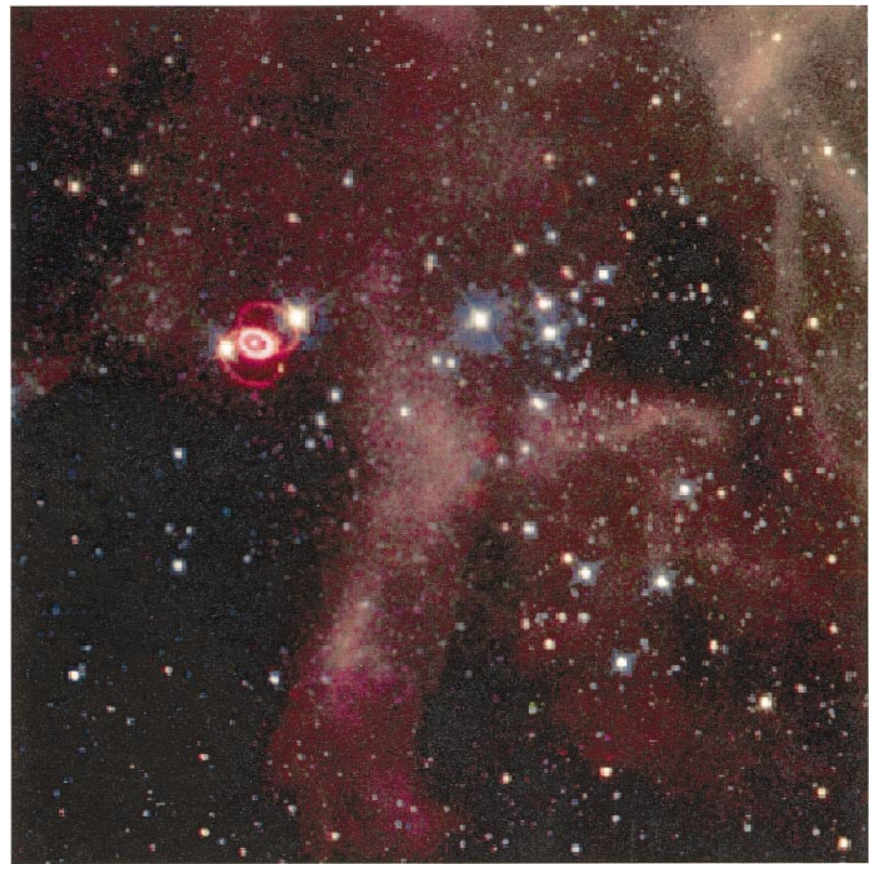

FIG. $7 a$

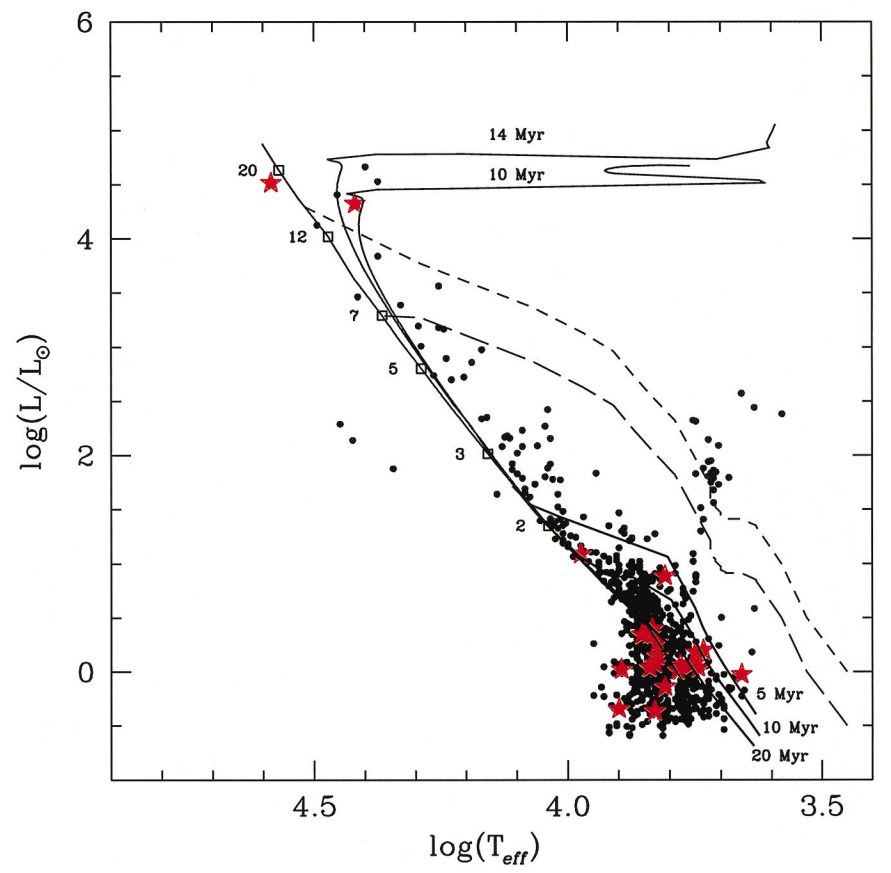

FIG. $7 b$

FIG. 7.- Properties of the central cluster. (a) Combined BVR plus H $\alpha$ image of an area 50" $\times 50^{\prime \prime}$ around SN 1987A. (b) Corresponding H-R diagram in

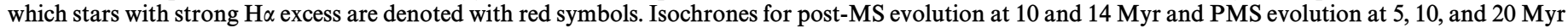
are also shown as are the birth lines for accretion rates of $10^{-4}$ (short-dashed line) and $10^{-5}$ (long-dashed line) $M_{\odot} \mathrm{yr}^{-1}$.

clusters in the LMC (7.7 $\pm 1.5 \mathrm{pc}$; Hodge 1988), suggesting that we are dealing with a "canonical" LMC cluster. Indeed, this group has been classified as a "loose cluster" by Kontizas, Metaxa, \& Kontizas (1988; their no. 80).

Efremov (1991) used Walker \& Suntzeff (1990) photometry to derive an approximate estimate of the cluster age of 10 Myrs and argued that SN 1987A was likely to belong to that cluster. Walborn et al. (1993) also used Walker \& Suntzeff (1990) photometry to discuss the properties of the cluster stars brighter than $V \simeq 20$. Adopting an average reddening of $E(B-V)=0.18$, they concluded that the cluster had an age of about $(12 \pm 4) \times 10^{6} \mathrm{yr}$.

As clearly seen in Figure $7 b$, with our data we can reduce the uncertainty and assign an age of $12 \pm 2 \mathrm{Myr}$ to the bulk of the stars present in this field. This age can fairly well account for the positions in the H-R diagram of both the most massive stars (with one exception; see below) and the PMS stars found in this field. There is one bright star $\left(\log T_{\text {eff }} \simeq 4.59, \log L / L_{\odot} \simeq 4.51\right)$ that appears to lie on the ZAMS and, therefore, to have a much younger age, say, 5 Myr or less. While statistical errors cannot account for this discrepancy, we notice that this star displays a significant $\mathrm{H} \alpha$ excess $\left(W_{\text {eq }} \simeq 12 \AA\right.$ ); therefore, it is one of the four Be stars we mentioned in $\S 4$. We argue that the strong $\mathrm{Be}$ characteristics are enough to alter the photospheric properties of this star so as to make it appear hotter and brighter than its average parameters would allow. In particular, if this is an almost pole-on Be star, we should expect its color temperature to be significantly higher than average. A "natural" consequence, which suggests a simple test to run, is that for a pole-on Be star the width of the $\mathrm{H} \alpha$ emission is expected to be relatively narrow.

Given the number of stars in the mass range 6-20 $M_{\odot}(15$ stars including the supernova progenitor), and adopting an IMF with slope $\Gamma$ in the range -1 to -2 , we estimate that the cluster originally included additional $2 \pm 1$ stars in the mass range $20-80 M_{\odot}$, which exploded as SN II well before the SN 1987A event. However, there is no clear sign of any shellike structure centered on the cluster. This implies that either the upper mass cutoff for this cluster was much lower than normal, i.e., no star more massive than $\sim 20 M_{\odot}$ formed in this cluster, or the more massive stars gave origin to explosions involving considerably lower kinetic energies than the canonical value of $10^{51}$ ergs.

The supernova is not at the center of the cluster; rather, it is offset about $15^{\prime \prime}$ to the east relative to the massive star barycenter, (i.e., the center of mass of 14 stars more massive than $6 M_{\odot}$ plus Sk -69202 itself). We speculate that this lack of "prominence" for the supernova progenitor may indicate that the few cluster stars that were more massive than the SN 1987A progenitor and exploded long ago were distributed over the cluster body in such a way as to fill the "gaps" in either the SN-star 2-star 3 group or in the southeast cluster extension (four massive stars), and the cluster center (eight massive stars).

\section{INITIAL MASS FUNCTION: A DISCUSSION}

As pointed out in $\S 5$, the number ratio of PMS low-mass (1-2 $M_{\odot}$ ) stars to massive stars in the central cluster is much smaller than the average in the whole field, i.e., 1.4 as compared to the average value 8.9 , indicating that there is an objective deficiency of low-mass stars in this young cluster. Such a deficiency would translate into an IMF slope that over the approximate mass range $1-14 M_{\odot}$ is considerably flatter than in the general field. In particular, if one adopts a slope $\Gamma=d \log N / d \log M \simeq-1.5$ for the entire field (see next section), the slope in the compact cluster would be approximately $\Gamma=-0.5$. On the other hand, a different conclusion would be reached if one limits the analysis to relatively high-mass stars. For example, if one 
assumes all stars brighter than $100 L / L_{\odot}$ to belong to one and the same stellar generation, the IMF between 3 and 14 $M_{\odot}$ would turn out to have a slope $\Gamma \simeq-1.2$, i.e., very similar to Salpeter's IMF. From these results one might conclude that a single power law cannot represent the observations and that the IMF agrees with Salpeter's for masses higher than $3 M_{\odot}$ and flattens dramatically for lower masses.

On the other hand, it is rather incautious to talk about an IMF when dealing with small number statistics and/or with very limited regions in space. In addition, looking at the cluster H-R diagram (cf. Fig. $7 b$ ), one can notice that many PMS stars appear to be appreciably older than $10 \mathrm{Myr}$, and actually appear to cluster around a $20 \mathrm{Myr}$ isochrone. This fact, while confirming that the most recent stellar generation is poor in low-mass stars, also tells us that the formation of lower mass stars in the cluster region is occurring on different timescales than that of more massive stars.

On a larger scale, the almost anticorrelation of the spatial distributions of high-mass and low-mass stars of a coeval generation (cf. $\S 5$ and Fig. 6) indicates that star formation processes for different ranges of stellar masses are rather different and/or require different initial conditions. An important corollary of this result is that the very concept of an "initial mass function" (IMF) may not have validity in detail but rather may be the result of a chaotic process, so it may make sense to talk about an average IMF over a suitably large area in which all different star formation processes are concurrently operating. Actually, if we just take the ratio of the total numbers of massive to low-mass stars belonging to the young population as reported in Table 2 and interpret them in terms of a power-law IMF adopting mass intervals of 6-15 $M_{\odot}$ and 1-2 $M_{\odot}$ for massive stars and for PMS stars, respectively, we would derive a slope for the initial mass function of $\Gamma=d \log N / d \log M \simeq-1.3$ with a purely statistical uncertainty of \pm 0.1 . Such a value is remarkably close to the classical Salpeter (1955) slope $\Gamma=-1.35$ for the solar neighborhood and is valid over essentially the same mass interval as Salpeter's original analysis, i.e., $\sim 2-10 M_{\odot}$. However, this is a conclusion drawn without taking into account of possible incompleteness effects. On the one hand, for both massive and lowmass stars above $\sim 1 M_{\odot}$, incompleteness due to missed detection and/or to crowding/blending is a negligible effect because all of these stars are well above our detection limit and their surface density in not so high (one star per $\sim 170$ WF pixel area, or, equivalently, an average separation of stars of $\sim 13$ WF pixels). On the other hand, for PMS stars one has to bear in mind that we can reliably identify only strong-line T Tau stars and that the total number of PMS stars of comparable masses may be considerably larger. For example, Alcalá et al. (1996) have shown that in the Orion region the number of weak-line $\mathrm{T}$ Tau stars is at least comparable to and possibly larger than that of strong-line $T$ Tauri stars. Actually, if one would identify PMS stars on the basis of their UV excess, i.e., as stars with much bluer $U-B$ colors than expected for normal stars with their observed $B-V$ and $V-I$ colors, one would count in our field as many as $\sim 850$ PMS stars (Romaniello 1998), i.e., a factor of 1.7 more than the positively identified T Tau stars. Moreover, $\mathrm{T}$ Tau stars are known to exhibit strong $\mathrm{H} \alpha$ variability on short timescales (e.g., Smith et al. 1999, and references therein). As a consequence, at any one time one may be able to detect only a fraction of the entire population of strong- line T Tau stars. Indeed, preliminary comparisons of overlapping regions in fields imaged at two different epochs (about 15\% of the entire field around SN 1987A) have shown that, while the number of strong-line $\mathrm{T}$ Tau stars identified at any one time is essentially constant, no more than one-half of the candidate T Tau stars display a significantly strong $\mathrm{H} \alpha$ excess at both epochs (Romaniello \& Panagia 2000, in preparation). This suggests that the total number of PMS stars may easily be twice as high as the number of strong-line $\mathrm{T}$ Tau stars we have identified, and possibly even higher. In turn, this result implies a significantly steeper IMF slope, say, $\Gamma \simeq-1.7$ or even more negative. A detailed discussion of these effects is presented in Romaniello (1998). Eventually, combining observations of the SN 1987A field taken at substantially more epochs than available at present, we will be able to properly identify all $\mathrm{T}$ Tau stars in the field so as to obtain a reliable statistics of the young population in the neighborhood of SN 1987A and to reach firm conclusions about star formation processes and history in this region of the LMC (Romaniello \& Panagia 2000, in preparation).

It is important to realize how crucial it is to identify and characterize each and every one of the PMS stars individually if one wants to evaluate an IMF reliably. For example, in our field we can identify about 500 PMS stars using an $\mathrm{H} \alpha$ excess criterion, and possibly almost 1000 on the basis of a UV excess. Still, such numbers represent a small fraction of the total number of stars with temperatures and luminosities comparable to those of the candidate PMS stars. Actually, limiting ourselves to stars that in the H-R diagram fall between the 5 and 40 Myr PMS isochrones and that are brighter than $L_{\odot}$ (i.e., for stars whose statistics is pretty complete), the ratio of the total number of stars to the number of bona fide PMS stars is $\sim 20$ if we include all possible candidates, and more than 40 if we include only strong-line T Tau stars. This means that in the absence of an explicit characterization of PMS stars, using just the location of stars in the H-R diagram as the criterion to recognize PMS stars will unavoidably introduce some heavy contamination. One can argue that, for a fixed surface density of old population stars, such an effect is much reduced when studying regions containing large concentrations of young stars. For instance, the possible contamination by older populations could be, say, $10 \%$ or less if the surface density of young stars in a given region were higher than 200-400 times the density of young stars around SN 1987A or, equivalently, 10 times the density of old population stars in the SN 1987A vicinities. However, since the density of old population stars with luminosities in the range $1<L / L_{\odot}<10$ is about 0.2 stars $\operatorname{arcsec}^{-2}$, exceeding that density by a factor of 10 , at least, would imply an average density of young low-mass stars $1<$ $L / L_{\odot}<10$, higher than two stars $\operatorname{arcsec}^{-2}$ and at least 10 times higher for stars in the next 1 dex bin in luminosity. It is easy to realize that with such high stellar densities another problem is bound to arise, at least for low-mass stars, namely, confusion/blending due to crowding. These effects will both "drown" faint stars into a "sea" of even fainter stars and/or would artificially create brighter stars by confusing nearby stars into one more luminous, apparently pointlike source (Panagia 2000, in preparation).

From this discussion, it follows that it is essential to have spectroscopic criteria that allow one to discern PMS stars from field stars unambiguously and completely. $\mathrm{H} \alpha$ excess 
and/or UV excess are possible ways of accomplishing this goal, but even these methods need confirmation, calibration and sharpening, in that we still have to compare our multiband photometry (or "wideband spectroscopy") with real spectra before a $100 \%$ reliable identification of $\mathrm{T}$ Tau stars can be claimed. We are going to fill this gap by taking medium- to high-resolution spectra of a number of our best T Tau candidates with the ESO-VLT1 in 2000 January (Favata et al. 2000, in preparation). With that it will eventually be possible to start talking about low-mass star formation and the low end of the IMF in the LMC on firm scientific grounds.

\section{CONCLUSIONS}

We have analyzed the WFPC2 images obtained in the $\mathrm{UV}, U, B, V, R, I$ broadbands and in the [O III] and $\mathrm{H} \alpha$ narrowband filters of the $130^{\prime \prime}$ radius region centered on $\mathrm{SN}$ 1987A, focusing on the properties of the stellar populations present in the field. The main results can be summarized as follows.

1. Over the entire field, we identify 21,995 stars, of which 6695 have overall photometric accuracy in the optical bands better than $0.1 \mathrm{mag}$.

2. From a comparison of the available six broadband photometry with model atmospheres, we have determined effective temperatures and bolometric luminosities for all of the 21,995 stars. For a subset of 2510 we also determined individual reddening corrections. Overall, we obtained an excellent characterization of a large fraction of the sample stars. In particular, the effective temperature was determined with an uncertainty lower than 0.05 dex in $\log \left(T_{\text {eff }}\right)$ for 9474 stars, most of which are brighter than $L / L_{\odot}=1$.

3. From an analysis of the resulting H-R diagram we have found evidence for a series of star formation episodes spanning from several billion years ago until at least 1-2 Myr and, possibly, still ongoing.

4. We have identified a generation of stars with ages about 10-14 Myr, which makes them coeval to the supernova progenitor and may include up to $35 \%$ of the young population in the entire field (defined as stars with ages less than $100 \mathrm{Myr}$ ).

5. The properties of the field old population indicates strong and continued star formation over a broad interval of $0.6-6 \mathrm{Gyr}$.
6. We have identified 488 strong-line $\mathrm{T}$ Tauri stars on the basis of their conspicuous $\left(W_{\mathrm{eq}}>8 \AA\right) \mathrm{H} \alpha$ excesses. Their ages peak at about 10-20 Myr and range from about $1 \mathrm{Myr}$ up to possibly $40 \mathrm{Myr}$. Their positions in the H-R diagram appear to require that star formation in the LMC occur with accretion rates about 10 times higher than in the Milky Way, i.e., $\sim 10^{-4} M_{\odot} \mathrm{yr}^{-1}$. This constitute the first positive detection of low-mass (about 1-2 $M_{\odot}$ ) PMS stars outside the Milky Way.

7. SN 1987A appears to belong to a loose, young cluster $12 \pm 2 \mathrm{Myr}$ old, in which the slope of the present mass function is almost identical to Salpeter's, i.e., $\Gamma \simeq-1.25$ for masses above $3 M_{\odot}$, but becomes much flatter for lower masses, i.e., $\Gamma \simeq-0.5$.

8. A comparison of the spatial distributions of massive stars and low-mass PMS stars shows that they are conclusively different, which indicates that different star formation processes operate for high- and low-mass stars.

9. We have addressed the problem of determining an IMF in our field. Because of the substantial differences of the spatial distributions of stars of different masses in our field, we conclude that the very concept of an IMF is not valid on a small scale (say, less than $10 \mathrm{pc}$ diameter-i.e., a scale comparable to the size of SN 1987A cluster), but it may become meaningful on a larger scale. A preliminary analysis shows that, averaging over the whole region (about 60 pc diameter), the IMF slope is steeper than Salpeter's IMF, i.e., $\Gamma \simeq-1.7$.

10. Since even the young stellar population is a mixture of generations of stars with different ages, we have concluded that a study of the IMF requires a proper separation of all of the various stellar populations coexisting with each other. We have shown that a proper characterization of the low-mass end of the IMF requires the explicit identification of individual PMS stars.

S. S. acknowledges the kind hospitality of STScI, where most of this work was done, as well partial support from the STScI Visiting Scientist Program. The comments of an anonymous referee were valuable in improving the presentation. This work was supported in part by HST-STScI grants GO-6437, GO-7434 GO-7821, GO-8243 to the SINS project (principal investigator is R. P. K.), and by STScIDDRF grants 82131,82160 , and 82186 to N. P.

\section{REFERENCES}

Alcalá, J. M., et al. 1996, A\&AS, 119, 7

Beaulieu, J. P., et al. 1966, Science, 272, 995

Bessel, M. S., Castelli, F., \& Plez, B. 1998, A\&A, 333, 231 (erratum 337, 321)

Biretta, J. A. 1996, WFPC2 Instrument Handbook 4.0 (Baltimore: STScI)

Brocato, E., \& Castellani, V. 1993, ApJ, 410, 99

Burrows, C. J., et al. 1995, ApJ, 452, 680

Cassisi, S., Castellani, V., \& Straniero, O. 1994, A\&A, 282, 753

Efremov, Yu. N. 1991, Soviet Astron. Lett., 17, 173

Fernandez, M., Ortiz, E., Eiroa, C., \& Miranda, L. F. 1995, A\&AS, 114, 439

Frasca, A., \& Catalano, S. 1994, A\&A, 284, 883

Gilliland, R. L. 1994, ApJ, 435, L63

Gilmozzi, R. 1990, Core Aperture Photometry with the WFPC (STScI Instrum. Rep. WFPC-90-96; Baltimore: STScI)

Gilmozzi, R., Kinney, E. K., Ewald, S. P., Panagia, N., \& Romaniello, M. 1994, ApJ, 435, L45

Herbig, G. H. 1960, ApJS, 4, 337

Herbig, G. H., \& Bell, K. R. 1988, Lick Obs. Bull. 1111

Hodge, P. W. 1988, PASP, 100, 1051

Hunter, D. A., Shaya, E. J., Holtzman, J. A., Light, R. M., O’Neill, E. J., \&

Lynds, R. 1995, ApJ, 448, 179

Jakobsen, P., et al. 1991, ApJ, 369, L63
Jaschek, M., Hubert-Delplace, A. M., Hubert, H., \& Jaschek, C. 1980, A\&AS, 42, 103

Kontizas, E., Metaxa, M., \& Kontizas, M. 1988, AJ, 96, 1625

Lamers, H. J. G. L. M., Beaulieu, J. P., \& de Wit, W. J. 1999, A\&A, 341, 827

Lucke, P. B., \& Hodge, P. W. 1970, AJ, 75, 171

Palla, F., \& Stahler, S. W. 1993, ApJ, 418, 414

Panagia, N. 1999, in IAU Symp. 190, New Views of the Magellanic Clouds, ed. Y.-H. Chu, N. Suntzeff, J. Hesser, \& D. Bohlender (San Francisco: ASP), 549

Panagia, N., Gilmozzi, R., Macchetto, F. D., Adorf, H.-M., \& Kirshner, R. P. 1991, ApJ, 380, L23

Panagia, N., Scuderi, S., Gilmozzi, R., Challis, P. M., Garnavich, P. M., \& Kirshner, R. P. 1996, ApJ, 459, L17

Romaniello, M. 1998, Ph.D. thesis, Scuola Normale Superiore, Pisa

Romaniello, M., Salaris, M., Cassisi, S., \& Panagia, N. 2000, ApJ, 530, 738

Salpeter, E. 1955, ApJ, 121, 161

Scuderi, S., Panagia, N., Gilmozzi, R., Challis, P. M., \& Kirshner, R. P. 1996, ApJ, 465, 956

Siess, L., Forestini, M., \& Dougados, C. 1997, A\&A, 324, 556

Smith, K. W., Lewis, G. F., Bonnel, I. A., Bunclark, P. S., \& Emerson, J. P. 1999, MNRAS, 304, 367 
Van Dyk, S., Hamuy, M., \& Mateo, M. 2000, in ASP Conf. Ser., SN 1987A: Ten Years After, ed. M. Phillips \& N. B. Suntzeff (San Francisco: ASP), in press

Walborn, N. R., Philips, M. M., Walker, A. R., \& Elias, J. H. 1993, PASP, 105,1240
Walker, A. R., \& Suntzeff, N. B. 1990, PASP, 102, 131

Whitmore, B. 1995, in Calibrating Hubble Space Telescope: Post-Servicing Mission, ed. A. Koratkar \& C. Leitherer (Baltimore: STScI), 269 